\title{
A multiperiodal management method at user level for storage systems using artificial neural network forecasts
}

\author{
G. Belli, G. Brusco, A. Burgio, D. Menniti, Member, IEEE, A. Pinnarelli, Member, IEEE, \\ N. Sorrentino, Member, IEEE, and P. Vizza, Student Member, IEEE
}

\begin{abstract}
The increase of renewable non-programmable production and the necessity to locally self-consume the produced energy led to utilize ever more storage systems. To correctly utilize storage systems, an opportune management method has to be utilized. This paper implements a multi-period management method for storage systems, using different management strategies. The method aims to minimize the total absorbed and supplied energy or the peak power exchanged with the grid. The results show the effectiveness of the method in diminishing the energy exchanged with the grid and also the possibility to optimize the performance of the storage systems.
\end{abstract}

Keywords - Storage management, multi-pediod scheduling, prosumer, optimal energy management

\section{INTRODUCTION}

$\mathrm{T}$ he common tendency to produce on site the necessary energy to the end user by means of small size plants, generally from non-programmable renewable sources, led to a rapid increase of installed photovoltaic (PV) power. Although PV generation offers economic and environmental benefits, its non-programmability can reduce these benefits. To increase the possibility to self-consume the produced energy and increase the profit for the user, storage systems should be utilized.

Otherwise, it is worth to underline that storage systems have a limited lifetime, related to their charge and discharge cycles. For this reason, it is opportune to manage storage systems with a specific strategy that can ensure them a long lifetime [1-2].

In order to better manage storage systems, realizing an accurate scheduling, generation and load forecasting systems would be useful to help the management of the grid.

Several methods carrying out storage management, considering different strategies, have been proposed in literature [3-7]. Such methods focus on an economical optimization, or a real time management without considering

This work was financed by the Italian Ministry of Economic Development (MISE) and the Ministry of Education, University and Research (MIUR) through the National Operational Program for Development and Competitiveness 2007-2013, Project DOMUS PON 03PE_00050_2.

G. Belli, G. Brusco, A. Burgio, D. Menniti, A. Pinnarelli, N. Sorrentino, and P. Vizza are with Department of Mechanical, Energy and Management Engineering (DIMEG) University of Calabria Via Bucci 42C, Arcavacata di Rende - CS, Italy (e-mail: name.surname@unical.it). the optimization of storage lifetime. In [3] the "economical" optimal management of a storage system is carried out in a single period ( 24 hours ahead); if the optimization is not required $24 \mathrm{~h}$ ahead, the method utilizes a real time approach. In [4] an appropriate management to reduce losses and increase distribution grid capacity is implemented and to this aim, a distributed storage is utilized; such a method allows to improve the utilization of renewable energy resources minimizing the energy adsorption from the grid.

Both methods $[3,4]$ are implemented on a $24 \mathrm{~h}$ period and they consider an economical optimization, moreover a multigeneration/multi-storage scheduling is realized.

It would be interesting to observe the benefits for a single user equipped with a PV generation system and a storage system, if the considered period is greater than $24 \mathrm{~h}$ and a multi-period optimal management method is adopted.

In [5] users equipped of energy storage systems are considered; it explains how the interaction of different storage systems can be harmful for the grid, although such storage systems have been introduced to protect it. So a novel management technique for distributed storage systems is implemented. The utilization of this technique led to saving up to $13 \%$ of the electricity bill for each consumer with a $4-\mathrm{kWh}$ storage system.

In [6] a day ahead optimization algorithm is implemented to provide the optimal storage and/or production scheduling strategy for a single user. The real load profile is considered exactly as the programmed $24 \mathrm{~h}$ ahead profile.

At the user level, that is a prosumer level, it is advantageous to know generation and load profiles forecast to choose the better storage power scheduling.

Indeed, a goal for the user should be to make "himselfsustainable", satisfying his total energy demand by means of local energy production, minimizing the exchanges of energy with the grid.

This paper presents a multi-period storage management method and considers a prosumer equipped with a storage system. In particular basing on weather forecast, generation and load forecasts are obtained; Artificial Neural Networks are utilized to implement the two forecasting models. Starting from these load and generation forecasts, different simulations are performed: the method minimizes the overall energy exchanged with the grid, the power peaks between prosumer 
and grid or the energy in a particular time interval; moreover, a limit for the power exchanged with the grid is considered. Worth noting that the benefits of the storage systems have been also demonstrated by the same authors in [8]; in particular, they underline the suitability of the Li-Ion batteries compared with lead-acid batteries. Indeed, in this paper the economic aspects concerning the storage systems and in particular the management of the storage system are not examined. In this paper, an energetic and power analysis is carried out.

The rest of the paper is structured as follows: in the second section, the implemented forecasting models are illustrated; in the third part, Storage Management Method is described; in the last part, simulation results are shown and the benefits in the use of the method are underlined.

\section{PV AND LOAD FORECASTING MODELS}

The Multi-Period Storage Management (MPSM) Method has for input the renewable production profile and the load profile of a user. To generate these profiles, the MPSM method uses renewable production and load forecasting models. Several production and load forecasting models exist in literature; they present different accuracy which depends on several factors, such as utilized input data, utilized methodology, and so on. Independently from the used method, the results of the generation and load power forecast can be evaluated in different way.

\section{A. Accuracy Estimation}

Many performance parameters are used according to forecast purpose. In general, all these parameters represent the error between the forecasted and the actual value.

The Mean Absolute Error (MAE) [9] measures how close forecasts are to the outcomes. It is more sensitive to high value of the discrepancy between the forecasted and the actual power profiles, so it is used to underline the presence of discrepancy peaks (emphasizing the high error). It allows to know the consequent average power imbalance; the MAE is given by:

$$
\text { MAE }=\frac{1}{N} \sum_{i=1}^{N}\left|f_{i}-y_{i}\right|=\frac{1}{N} \sum_{i=1}^{N}\left|e_{i}\right|
$$

where $\mathrm{fi}$ is the forecast value, yi is the real value. Moreover, the evaluation of the Maximum Absolute Error (MaxAE) is important to know the maximum difference between fi and yi, in particular it is useful to know the maximum power imbalance resulting from the forecast; it is given by:

$$
\text { MaxAErr }=\operatorname{Max}\left(\left|f_{i}-y_{i}\right|\right)
$$

The Mean Squared Error (MSE) [9] measures the mean square discrepancy between fi and yi. The MSE is more sensitive than the MAE to high error value. The MSE square root provides another statistical quantity, that is the Root Mean Squared Error (RMSE). The MSE and the RMSE are defined as:

$$
M S E=\frac{1}{N} \sum_{i=1}^{N}\left(\mathrm{f}_{\mathrm{i}}-\mathrm{y}_{\mathrm{i}}\right)^{2}
$$

$$
R M S E=\sqrt{M S E}=\sqrt{\frac{1}{N} \sum_{i=1}^{N}\left(\mathrm{f}_{\mathrm{i}}-\mathrm{y}_{\mathrm{i}}\right)^{2}}
$$

Another important statistical quantity is the Mean Absolute Percentage Error (MAPE) [9]. It measures the forecasting accuracy and expresses this accuracy as a percentage. It is calculated by:

$$
M A P E=\frac{1}{N} \sum_{i=1}^{N}\left|\frac{\mathrm{f}_{\mathrm{i}}-\mathrm{y}_{\mathrm{i}}}{\mathrm{f}_{\mathrm{i}}}\right|
$$

Moreover, the Maximum Percentage Error (MAxPE) allows knowing what the maximum percentage discrepancy between the forecasted and the actual value is:

$$
\operatorname{MaxPE}=\max \left(\left|\frac{f_{i}-y_{i}}{f_{i}}\right|\right) \quad \forall i=1 \ldots N(6)
$$

Such statistical parameters allow to estimate the accuracy of the several forecasting models, to find the best suited to the case under estimation.

\section{B. PV forecasting model}

Referring to renewable production forecasting model, it is supposed that the power source is a photovoltaic (PV) plant.

Several PV forecasting models exist in literature: they often present accurate results. However, such models are very sophisticated and require information not always available, as those presented in [10-12]; the PV forecasting model implemented by the authors uses available input data and for this reason it is defined a "practical" forecasting model.

Such a PV generation forecasting model is implemented by an Artificial Neural Network (ANN).

For the implementation of the ANN, the Neural Network Toolbox of Matlab is utilized. The chosen ANN typology is a Multi-Layer Perceptron (MLP), with a supervised training algorithm; in particular, the back-propagation algorithm is used. Moreover, only a hidden layer is utilized, the activation function of all the neurons is tan-sigmoidal, while the training function is the Levenberg-Marquardt method [13].

Chosen the ANN typology, it is necessary to provide to the method historical input data and historical output (target) data (in the case under examination the collect of the PV power production data really generated by the PV plant).

The number of neurons of the input layer is chosen considering the input data, while the number of neurons of the hidden layer has been determined empirically. In fact, a sensitivity analysis has been conducted: the input data are kept constant and the number of neurons of the hidden layer are changed; under this condition, several tests to calculate the MAPE have been made. The optimum number of neurons for the hidden layer, to minimize the MAPE is 30 , as shown in Figure1. 


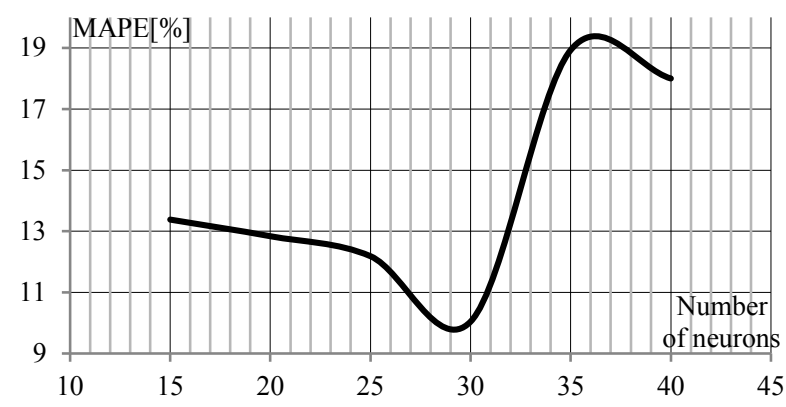

Fig. 1. Sensitivity analisys

So, summarizing, the implemented ANN consists in (Fig. 2):

- 5 input neurons (meteorological condition of the considered hour $\mathrm{h}$, meteorological condition of the hour $\mathrm{h}+1$, meteorological condition of the hour $\mathrm{h}-1$, the hourly irradiance, the considered hour);

- 30 hidden layer neurons;

- 1 output neuron (hourly forecasted power production).

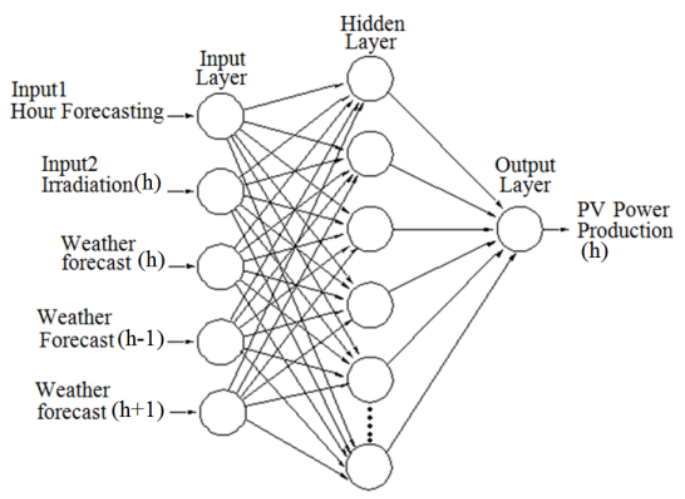

Fig. 2. ANN PV Forecasting Representation

Meteorological data about sky conditions are transformed to be used as input data for the ANN, in a number. A "1" to "5" scale has been used to represent the increasing of cloudiness. The minimum value ("1") indicates "clear-sky" condition while the maximum value ("5") indicates "storm" (Tab.1).

TABLE I. WEATHER CONDITIONS CODING

\begin{tabular}{|c|c|}
\hline Code & Weather condition \\
\hline 1 & Clear sky \\
\hline 2 & Nearly cloudless, scattered clouds \\
\hline 3 & Few clouds \\
\hline 4 & Partly cloudy \\
\hline 5 & Covered, Storm \\
\hline
\end{tabular}

The model has been tested on a specific a PV plant and good results are obtained both for clear and non-clear sky conditions.

Indeed, the results show that: in clear sky conditions the MAPE is less than $10 \%$, while in non-clear sky conditions the MAPE is about $42 \%$. The MAE, compared to PV power plant, is equal to $2.6 \%$ for clear sky conditions and $6.8 \%$ for nonclear sky conditions.
Figure 3 depicts the results of the PV forecast for the days from 30 August to 2 September; for these days, clear-sky conditions were predicted. The MAPE is calculated for all the four days: it is equal to $9.8 \%$. Whereas, the percentage error is maximum and equal to $45 \%$ in the hours close to sunrise and sunset; while the percentage error becomes minimum (less than $1 \%$ ) for the hours of higher production.

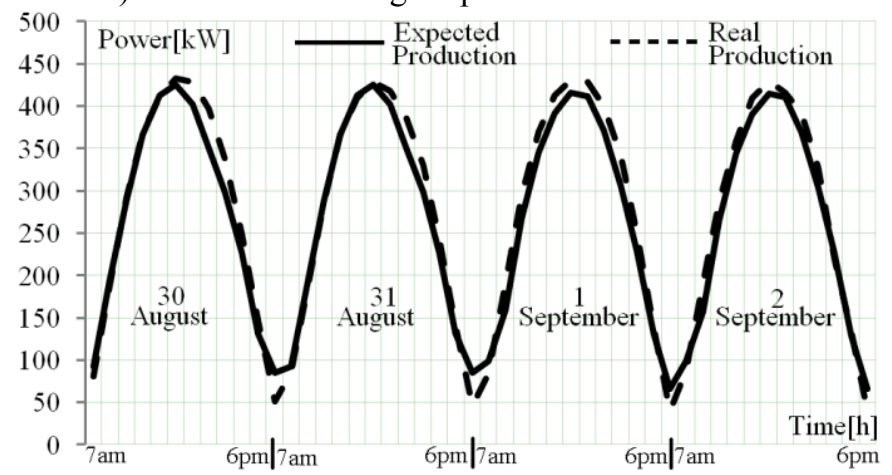

Fig. 3. Clear-Sky Days Results

\section{Load forecasting model}

Referring to the load forecasting model, in [14] the relevance to use load forecast for several purposes is underlined, especially for islanded operation, because it is necessary to guarantee grid stability, in addition to allow generation programmable system to work at a maximum efficient point.

In literature, there are a large number of load forecasting models as those presented in [15]. Reference [16] highlights that is more difficult to predict the individual load than an aggregate of loads. Nevertheless, in this paper a predictive model for an individual load is implemented.

The load forecasting model implemented in this paper utilizes accessible data; such a model predicts also individual and aggregate loads. Although its simplicity the results demonstrate the good performances of the model.

A feed-forward Multi-Layer Perceptron (MLP) ANN, supervised by a back-propagation algorithm, has been implemented (Fig. 4). For ANN training, the collection of consumption data of the considered user is necessary. Similarly than PV forecasting model, also for load forecasting model a sensitivity analysis has been carried out, so to detect the number of the hidden layer neurons which lead to a better accuracy of the model.

The implemented ANN consists in:

- 7 input neurons (month, day, day type, hour, daily maximum temperature, daily minimum temperature and daily average temperature);

- 30 hidden layer neurons;

- 1 output neuron (hourly forecasted power consumption). 


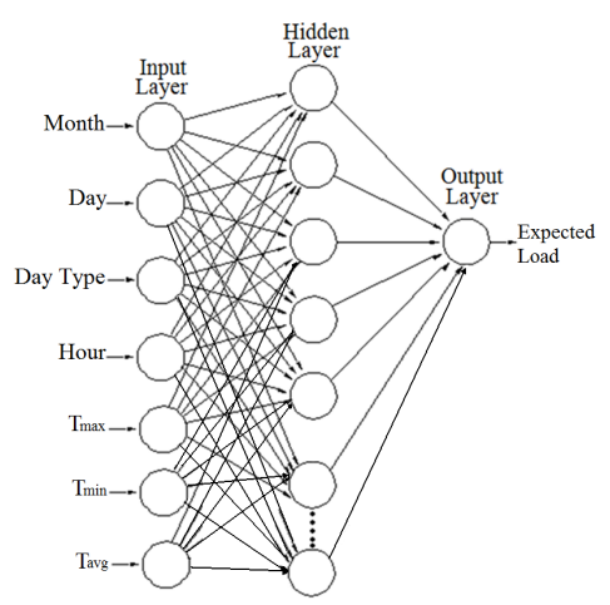

Fig. 4. ANN Load Forecasting Representation

The input data are so defined: the day, month and hour identify the period which the forecast is required; the day type identify if the considered day is a workday or a holiday, if it is a day before or after a holiday. Moreover, the minimum, maximum and average temperature are utilized; these are useful specially if the electric air conditioning is utilized, in particular maximum temperature is useful for cooling, whereas minimum temperature is useful for heating.

The load forecasting model has been tested on real data of a typical residential user. In Fig. 5, the forecasted and the real load profiles are shown, for three days (Thursday, Friday and Saturday). The obtained Mean Absolute Error (MAE), compared to the rated power of the considered user's contract is less than $6 \%$, that is an acceptable error for the purpose of the method.

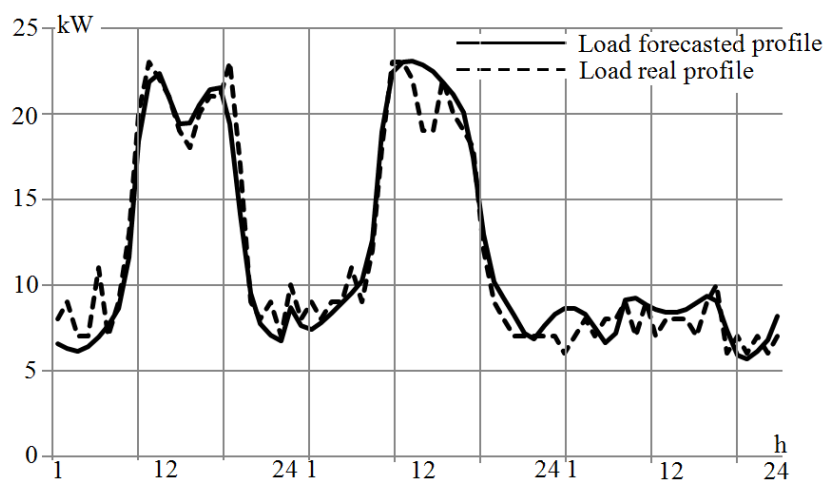

Fig. 5. Forecasted load profile and real load profile

Figure 5 depicts the results of the load forecast model; the MAPE is calculated and it is less than $20 \%$. Whereas, the percentage error is maximum in the hours and day with a low consumption, and the minimum percentage error occurs for the workdays, when the consumption is high, and it is less than $1 \%$.

\section{Storage Management Method description}

The most important variables used in the Multi-Period Storage Management (MPSM) method are reported in Table 2.

TABLE II. MPSM METHOD VARIABLES

\begin{tabular}{|c|l|}
\hline \multicolumn{2}{|c|}{ Nomenclature } \\
\hline$P_{g_{t}}^{d}$ & Grid Transferred Power; (time t, day d) \\
\hline$P_{L_{t}}^{d}$ & Load Power; (time t, day d) \\
\hline$P_{S_{t}}^{d}$ & Storage Transferred Power; (time t, day d) \\
\hline$E_{S_{t}}^{d}$ & Storage Energy Level; (time t, day d) \\
\hline$E_{S_{\min }}, E_{S_{\max }}$ & Minimum and maximum Storage Energy Level \\
\hline$P_{S_{\min }}, P_{S_{\max }}$ & Minimum and maximum Storage Power \\
\hline$E_{S_{\text {Init }}}$ & Initial Energy stored \\
\hline
\end{tabular}

The main equations which describe the MPSM method are as follow:

$$
O F: \min \left(\sum_{\substack{t=1 \\ d=1}}^{D} f\left(P_{g_{t}}^{d}\right)\right)
$$

s.t.

$$
\begin{gathered}
P_{g_{t}}^{d}=P_{L_{t}}^{d}-P_{P V}^{d}-P_{S_{t}}^{d} \\
E_{S_{t+1}}^{d}=E_{S_{t}}^{d}+P_{S_{t}}^{d} * \Delta t \\
E_{S_{\min }} \leq E_{S_{t}}^{d} \leq E_{S_{\max }} \\
P_{S_{\min }} \leq P_{S_{t}^{d}}^{d} \leq P_{S_{\text {max }}} \\
\operatorname{sign}\left(P_{S_{t}}^{d}\right)=\operatorname{sign}\left(P_{P_{t}}^{d}-P_{L_{t}}^{d}\right) \\
E_{S_{t=1}^{d=1}}^{d=}=E_{S_{\text {Init }}}
\end{gathered}
$$

The MPSM method can be utilized for more days (d) and every day is divided in more time intervals $(\mathrm{t})$; such time intervals are the same of that used in the forecasting models.

In Objective Function (OF) (7), $f\left(P_{g_{t}}^{d}\right)$ indicates different goals of energy exchange optimization. Indeed, the user can require to: minimize overall the energy exchanges with the grid, minimize the power peaks or minimize the energy exchanged for a particular time period with the grid

The MPSM method is subjected to the constraints from (8) to (13). Constraint (8) is used to calculate the power exchanged with the grid, $P_{g_{t}}{ }^{2}$. The constrains (9), (10), (11), (12) and (13) concern the storage. In (9) the variation of the stored energy (between two time intervals) is calculated, in (10) the stored energy is limited between a minimum and maximum value (depending on the used storage); in (11) the charge and discharge storage power is limited, (12) indicates that the storage can charge only if there is a power surplus (PV power $P_{P V}{ }_{t}^{d}$ is greater than the load power $P_{L_{t}}^{d}$ ), vice versa storage can only discharge. In (13) the initial stored energy is defined as $E_{S_{\text {Init }}}$.

Once load and PV production power forecasts for the user are obtained, the difference between the two profiles is calculated. This difference profile represents the input of the 
MPSM method, which solves the Objective Function (OF), taking into account the constraints. The method returns the storage power exchange profile and the consequent grid power exchange profile.

\section{SIMULATION}

To test the effectiveness of the MPSM method, some simulations are carried out, considering as prosumer a build of University of Calabria: this is a business user, equipped with photovoltaic plants.

The considered build has a maximum power consumption of $25 \mathrm{~kW}$ and the installed PV plants power is $45 \mathrm{~kW}$.

The test considers a time period of 7 days, from the $10^{\text {th }}$ to $16^{\text {th }}$ October 2015. In Fig. 6, load and PV power forecast profiles of the considered 7 days are reported.

After determining load and PV power profiles, it is necessary to sizing storage system for the required function.

\section{A. Non optimized PV power}

First of all, the storage capacity is calculated to supply loads and limit the exchange of energy with the grid.

Storage capacity will be the smallest between the resulting average daily energy purchased and supplied to the grid, which are calculated as the difference between PV production and load profiles. This analysis is carried out for profiles of a typical day. In the present case, the daily purchased energy is almost $170 \mathrm{kWh}$, whereas the energy supplied to the grid is 80 $\mathrm{kWh}$; so the storage would have a capacity of $80 \mathrm{kWh}$.

After calculating storage capacity, an overestimation to be conservative will be necessary: an increase of $20 \%$ will be considered. In addition, in order to safeguard the storage useful life, a residual state of charge (SOC) of $40 \%$ has to be considered as a further increase of the estimated storage capacity.

Considering the previous estimated storage capacity ( 80 $\mathrm{kWh}$ ) and the increases of $20 \%$ and $40 \%$, the obtained storage capacity is about $140 \mathrm{kWh}$.

Starting from the calculation of the difference between load and PV power forecasts, it is used for two groups of simulations. The first group of simulation aims to minimize

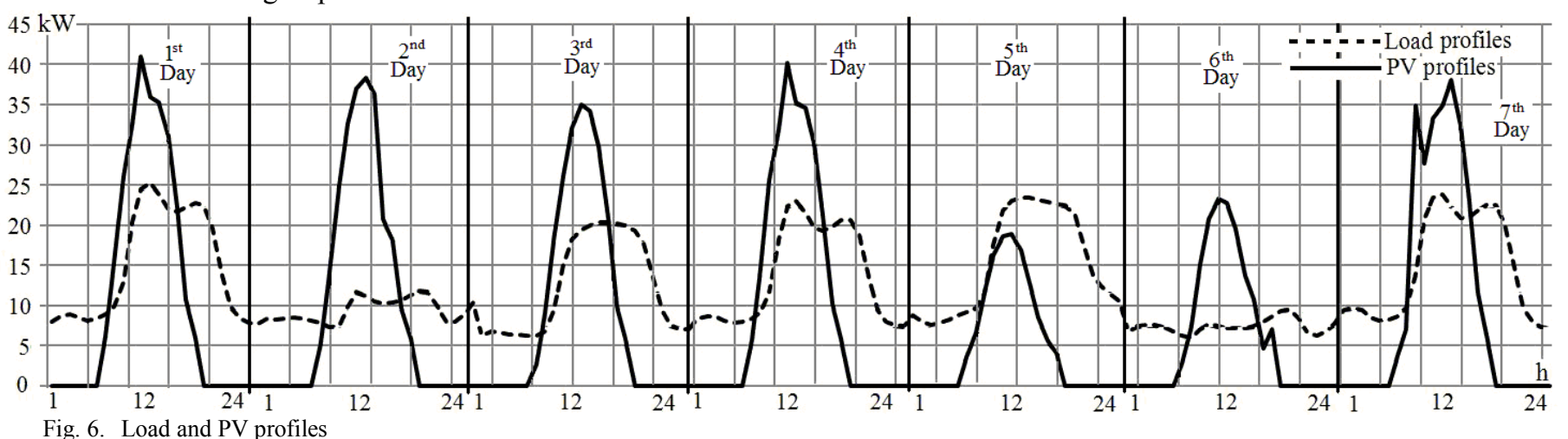

the total exchange of energy with the grid, trying to make the prosumer self-sustainable and to avoid congestions on the grid. Instead, the second group of simulations aims to minimize only the peaks of energy during the day, trying to reduce the costs of energy supply and to avoid worthless oversize of the generation plants. For both the groups of simulations, the comparison is made between the condition with storage working in "real time", that is no storage management is taking into account, and the condition with storage managed by MPSM method.

The starting point is the value of the overall energy exchanged between the user and the grid without using a storage system: this value is equal to $1.68 \mathrm{MWh}$, where 1.13 $\mathrm{MWh}$ is the energy adsorbed by the user and $0.55 \mathrm{MWh}$ is the energy left to the grid.

For the first groups of simulations, when none management method is utilized, the total energy exchange with the grid decreases until 0.69 MWh, where 0.60 MWh is the purchased energy by the user and $0.09 \mathrm{MWh}$ supplied to the grid. If the storage is managed by MPSM method, the total energy exchanged with the grid is equal to $0.68 \mathrm{MWh}$. Respect the previous case, the difference is very limited. Although this difference is only of $0.01 \mathrm{MWh}$, the positive effect of the MPSM method consists in the possibility to maximize the performances of the storage. Indeed, using a "real time" operation strategy, the charge and discharge cycles are not optimized because they are partial cycles, while with MPSM method, the storage executes always full cycles of charge and discharge (Fig.7). Only in a few hours, a distorted trend is visible in Fig. 7, due to the high variability of weather conditions on the $5^{\text {th }}$ day, that involves to have a partial cycle of charge and discharge.

For the second group of simulations, minimizing only the peaks of power exchanged with the grid, the power exchanged in "real time" reaches $23 \mathrm{~kW}$, while using MPSM method, it is about $8 \mathrm{~kW}$. In Fig. 8 the exchanged energy profile with and without MPSM method are depicted.
Fig. 6. Load and PV profiles 


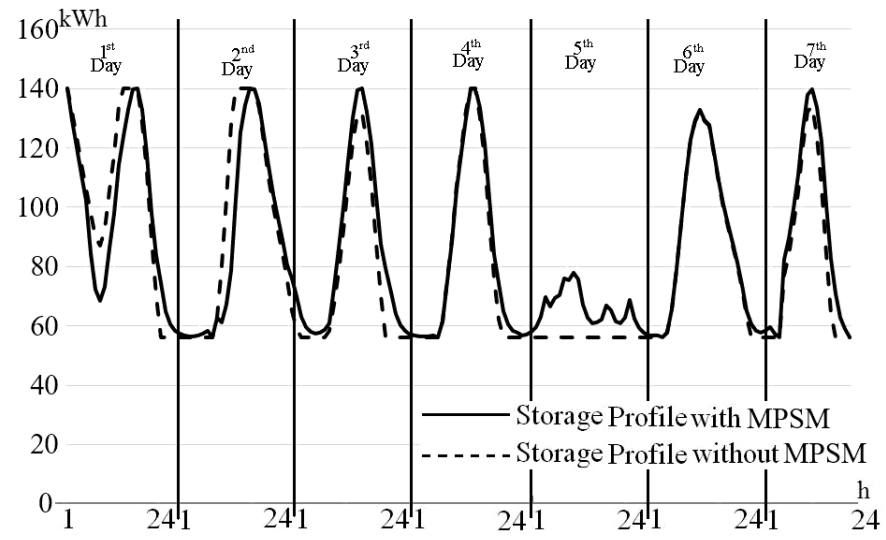

Fig. 6. Stored Energy Profile with and without MPSM

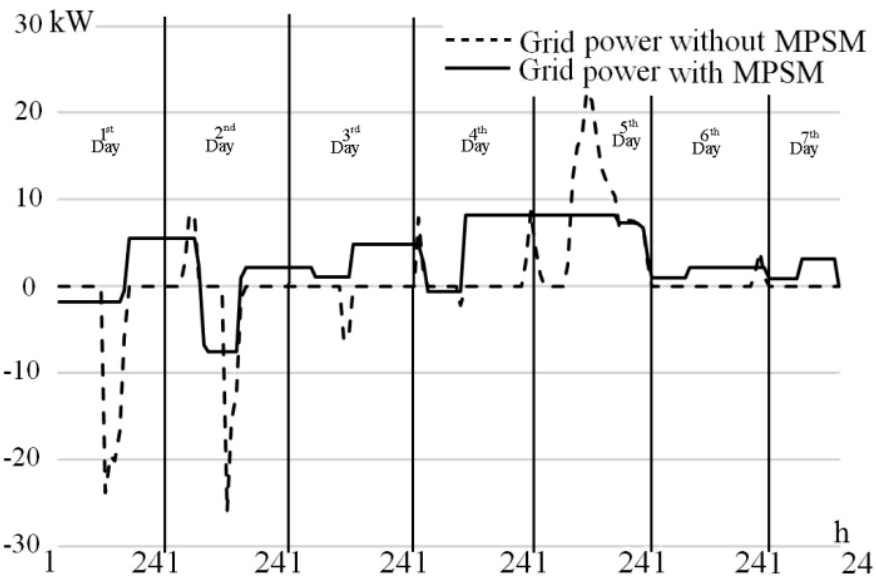

Fig. 7. Exchanged power profiles with and without MPSM

\section{B. Optimized PV power}

In this section, starting from the average daily load profile and the monthly average daily PV production profile, to minimize the exchange of energy with the grid, the PV plant is sized to cover the daily energy demand. Considering this, the obtained rated PV power is about $56 \mathrm{~kW}$.

Similarly to the previous subsection A, the storage system is properly sized and the obtained capacity is about $240 \mathrm{kWh}$. With such data the method is utilized to carry out the same test of the previous case.

First of all, the MPSM method is utilized to minimize the exchange of energy with the grid; the obtained result of the total energy exchanged with the grid is equal to $0.41 \mathrm{MWh}$, where $0.25 \mathrm{MWh}$ is the purchased energy by the user and 0.16 MWh is the energy supplied to the grid.

In this case, as the rated PV power and the storage capacity are optimized, the use of the MPSM method, compared to the real time management, does not contribute to many advantages in the management of the charge/discharge storage cycles.

The real advantage would occur in the management of the exchanged energy with the grid for the days with non-clear sky conditions. In Fig. 9 the exchanged energy profile with the grid, with and without the MPSM method is depicted.

Moreover, referring to Fig. 9, it is possible to observe that the energy supplied to the grid is greater than the energy purchased from the grid; only the $5^{\text {th }}$ day the purchased energy is greater than the supplied one, because it is not a clear sky day.

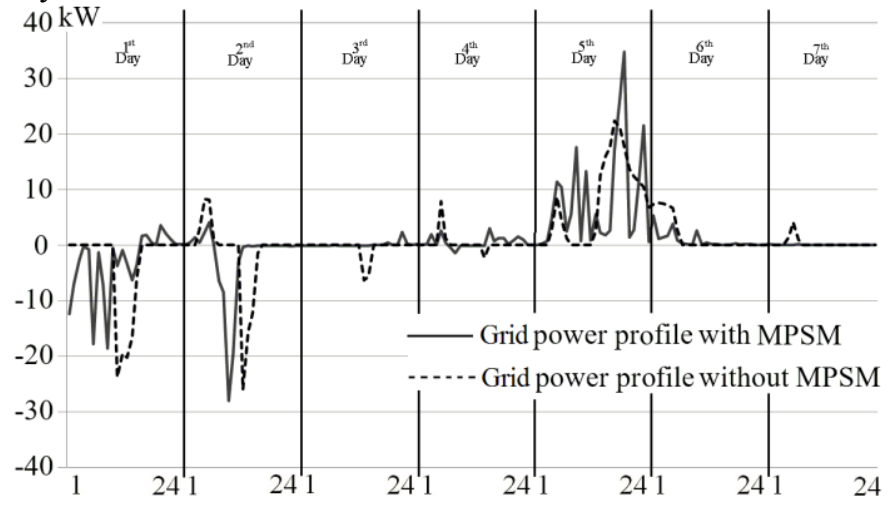

Fig. 8. Exchanged grid power profile with and without MPSM

Worth noting that the PV power is sized to cover the daily energy demand; so if the rated PV power increase, obviously the produced energy increase and as a consequence the total energy exchanged with the grid increases. In fact, for example, if the rated PV power is $60 \mathrm{~kW}$ the total exchanged energy is $0.49 \mathrm{MWh}$, instead of $0.41 \mathrm{MWh}$. This shows that before to use the MPSM method, optimal PV and storage sizing is necessary.

\section{Grid Power Restriction}

In this section, it is supposed that the considered prosumer has a limit for $P_{g_{t}}^{d}$. This can be due to different reason, for example if the prosumer has a contract with the energy provider for a reduced power, or if the power line is designed for a limited power.

In fact, this kind of optimization allows to reduce the problems due to the congestion problem and any restrictions of the power interface devices.

In particular two cases are examined: in the first case the maximum $P_{g_{t}}^{d}$ (Pg_max) is $10 \mathrm{~kW}$, in the second case Pg_max is $7 \mathrm{~kW}$. Worth noting that the limit for the power is both for the supplied and delivered energy. The MPSM method is completed using the sequent equations:

$$
\left|P_{g_{t}}^{d}\right| \leq P_{g_{-} \max }
$$

The $\mathrm{OF}$ is implemented to minimize the entire energy exchanged with the grid, as implemented above. It is worth to underline that this test is different to the previous minimization of the peak power, in fact in the previous case a restriction for $P_{g_{t}}{ }^{d}$ is not utilized but solely the peaks of $P_{g_{t}}{ }^{d}$ are reduced.

In the first test Pg_max is equal to $10 \mathrm{~kW}$ and the constraint (12) is relaxed, in this way the storage can be charged also by the grid and can discharge also if there is a surplus of energy, this is limited only through the OF.

The utilized storage capacity is $240 \mathrm{kWh}$ and the rated PV power is $56 \mathrm{~kW}$; the load profile is reported in Fig. 6.

In this first case, the obtained result of the total energy exchanged with the grid is equal to $0.41 \mathrm{MWh}$, where 0.26 $\mathrm{MWh}$ is the purchased energy by the user and $0.15 \mathrm{MWh}$ is the energy supplied to the grid. 
In the second case, Pg_max is equal to $7 \mathrm{~kW}$, the total energy exchanged with the grid is also equal to $0.41 \mathrm{MWh}$, and the purchased energy is equal to $0.26 \mathrm{MWh}$ whereas the supplied energy to the grid is equal to $0.15 \mathrm{MWh}$.

Such results demonstrate that the constrains on $P_{g_{t}}^{d}$ are almost irrelevant for the OF, in fact the quantity of energy exchanged with the grid is the same of the previous case. The only difference is for the profile of $P_{g_{t}}{ }^{d}$ : in Figs. 10 and 11 the profiles of power exchanged with the grid for either cases are reported.

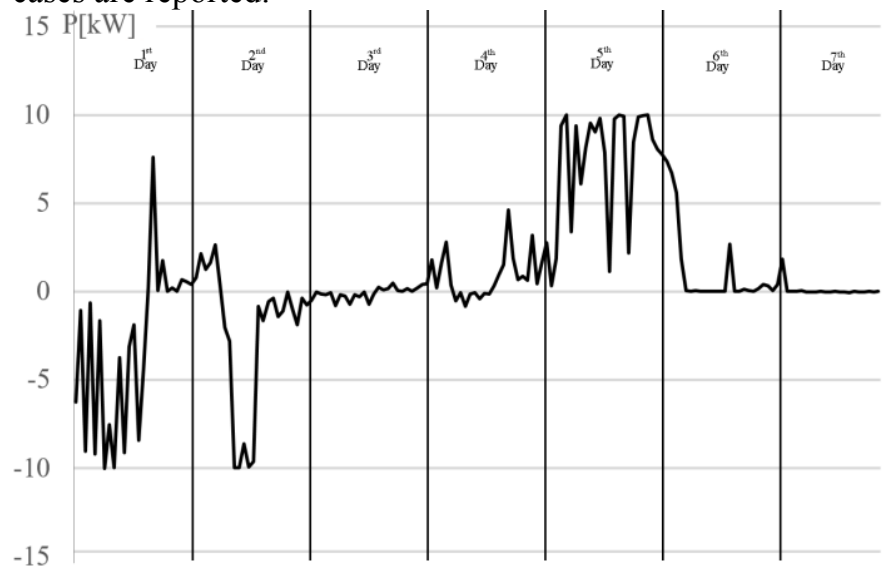

Fig. 9. Exchanged grid power profile with the constrain $P_{g_{\_} \max }=10 \mathrm{~kW}$

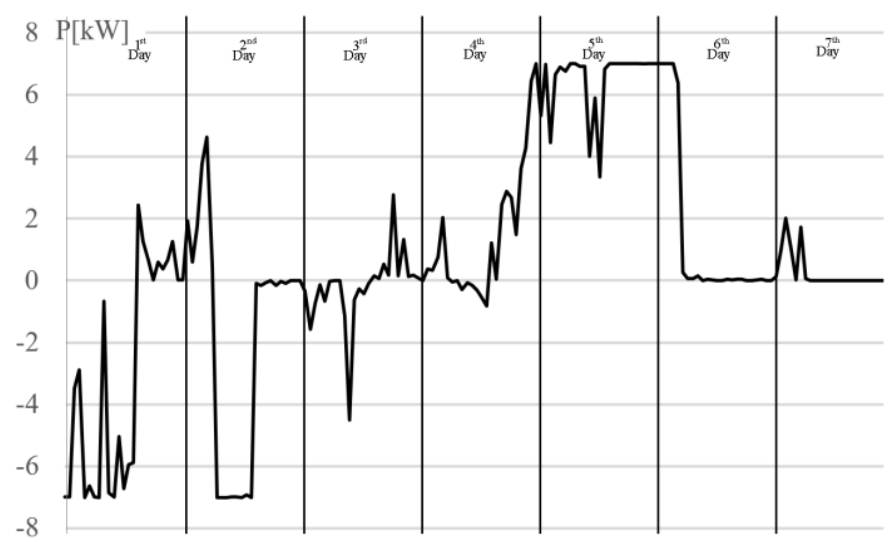

Fig. 10. Exchanged grid power profile with the constrain $P_{g_{\text {max }}}=7 \mathrm{~kW}$

The figures show that the trend of $P_{g_{t}}^{d}$ is constant in the area where the power peak occur, this means that for those time and day the bonds are achieved; this is particularly observable for Pg_max is $7 \mathrm{~kW}$.

Moreover, worth noting that the maximum power Pg_max $(7 \mathrm{~kW})$, obtained in this case, is less than the maximum power obtained with the minimization of the peak of power (in Subsection A) where Pg_max is $8.2 \mathrm{~kW}$.

It is important to observe the behaviour of the storage system when Pg_max is equal to $7 \mathrm{~kW}$ compared to the case when there is not a constrain for $P_{g_{t}}^{d}$ : in figure 12 this comparison is reported.

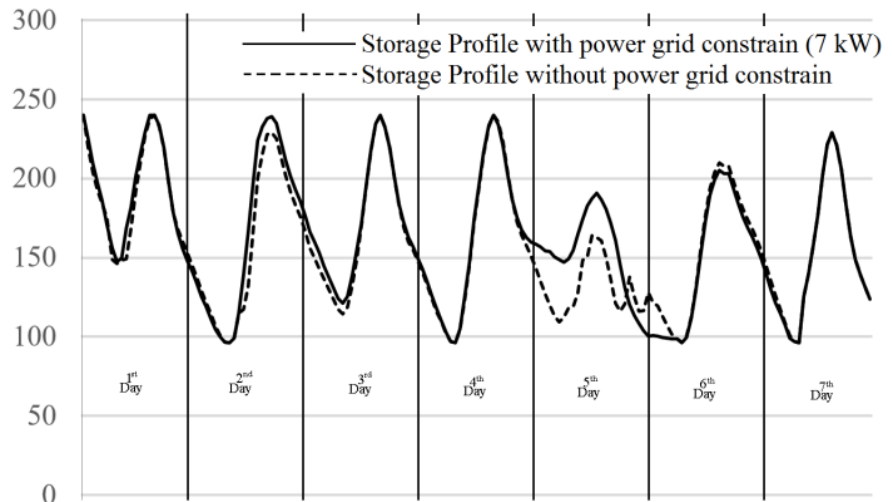

Fig. 11. Stored Energy Profile with and without $\mathrm{P}_{\mathrm{g} \_ \text {max }}$ constrain

It is possible to observe the differences between the two profiles, especially for the fifth day. In fact, to limit the power exchanged with the grid, in particular the power drawn from the grid, the stored energy is maintained as long as it is not used to decrease $P_{g_{t}}^{d}$ : the battery is not discharged just when there is a deficit of energy but when this energy is utilized to limit the maximum $P_{g_{t}}^{d}$.

Thanks to the MPSM method the prosumer can employ a reduced power contract with consequent less costs for the prosumer. At the same time the Distribution System Operator (DSO) can design the line for a reduced power, with further savings.

\section{CONCLUSION}

The paper shows the importance of an opportune management method for storage devices. In fact, the positive effect resulting from the use of storage systems, particularly in relation to non-programmable resources, can be increased if an appropriate management strategy is utilized. One of the feature of the implemented method is its multi-periodicity. In fact, if the management is made on more days, there are more data input and the storage can be managed in a better way.

The presented storage management method implements different management goals.

First of all, the method minimizes the total energy exchanged with the grid to make users self-sustainable. This implies the use of opportune PV and load forecast models. Secondly the method is also utilized to reduce the peak of power exchanged with the grid, decreasing from $23 \mathrm{~kW}$ to about $8 \mathrm{~kW}$.

Moreover, it is underlined that an accurate sizing of PV and storage systems is necessary before to implement and utilize a management strategy.

The results are compared with the real time storage management; such a comparison shows the effectiveness of the method. The results show also the possibility to optimize the performance of the storage device in terms of charge and discharge cycles.

At the end, the behaviour of the management method, if a constrain for the maximum $P_{g_{t}}^{d}$ is utilized, is evaluated. Simulations are carried out; they demonstrate that despite a further constrain is utilized, the entire energy exchanged with the grid is minimized. This can be a good result both for the 
prosumer and for the DSO, indeed they can respectively reduce the contract power and reduce the line capacity, with consequent savings.

Moreover, it would be interesting evaluate the behaviour of the method if a schedulable load is adopted.

\section{REFERENCES}

[1] D. Menniti, A. Pinnarelli, N. Sorrentino, A. Burgio, and G. Brusco, "Energy Management System for an Energy District With Demand Response Availability", Smart Grid, IEEE Transactions on, vol. 5(5), 2014, pp. 2385-2393.

[2] D. Menniti, A. Pinnarelli, N. Sorrentino, G. Belli, A. Burgio, "Demand Response Program in an Energy District with storage availability", International Review of Electrical Engineering, in press.

[3] A. Nottrott, J. Kleissl, and B. Washom, "Energy dispatch schedule optimization and cost benefit analysis for grid-connected, photovoltaicbattery storage systems", Renewable Energy, vol. 55, 2013, pp. 230-240.

[4] N. Jayasekara, P. Wolfs, and M.A.S. Masoum, "An optimal management strategy for distributed storages in distribution networks with high penetrations of PV", Electric Power Systems Research, vol. 116, 2014, pp. $147-157$.

[5] P. Vytelingum, T.D. Voice, S.D. Ramchurn, A. Rogers, and N.R. Jennings, "Agent-based micro-storage management for the smart grid", in Proc. of the 9th International Conference on Autonomous Agents and Multiagent Systems, vol. 1, International Foundation for Autonomous Agents and Multiagent Systems, pp. 39-46, May 2010.

[6] I. Atzeni, L.G. Ordóñez, G. Scutari,D.P. Palomar, and J.R. Fonollosa, "Demand-side management via distributed energy generation and storage optimization", Smart Grid, IEEE Transactions on, vol. 4(2), 2013, pp. 866-876.

[7] A. Mohamed, and O. Mohammed, "Real-time energy management scheme for hybrid renewable energy systems in smart grid applications", Electric Power Systems Research, Vol. 96, 2013, pp. 133-143.

[8] D.Menniti, A. Pinnarelli, N. Sorrentino, A. Burgio, G. Brusco, "The economic viability of a feed-in tariff scheme which solely awards the self-consumption for promoting the use of integrated photovoltaicbattery systems", Applied Energy, in press.

[9] S. Makridakis, and M. Hibon, "Evaluating accuracy (or error) measures", Working paper 95/18/TM, INSEAD, (1995) France.

[10] Y. Zhang, M. Beaudin, Raouf Taheri, H. Zarcipour, and D. Wood, "Day-Ahead Power PV power production Output Forecasting for Small Scale Soar Photovoltic Electricity Generators", IEEE Transactions on Smart Grid, Vol. 6, no. 5, September 2015

[11] C. Chen, S. Duan, T. Cai, and B. Liu, "Online 24-h solar power forecasting based on weather type classification using artificial neural network", Solar Energy, Vol. 85, no. 11, 2011, pp. 2856-2870.

[12] C. W. Chow, B. Urquhart, J. Kleissl, M. Lave, A. Dominguez, J. Shields, and B. Washom, "Intra-hour forecasting with a total sky imager at the UC San Diego solar energy testbed", Solar Energy, Vol 85, no. 11, 2011, pp 2881-2893.

[13] J. J. Moré, "The Levenberg-Marquardt algorithm: Implementation and theory", in Lecture Notes in Mathematics, No. 630-Numerical Analysis, Springer-Verlag, 1978, pp. 105-116.

[14] N. Hatziarg, Microgrids: Architectures and Control, Wiley-IEEE Press, February 2014.

[15] H. S. Hippert, C. E. Pedreira, and R.C. Souza, "Neural networks for short-term load forecasting: A review and evaluation", Power Systems, IEEE Transactions on, vol. 16(1), 2011, pp 44-55.

[16] H. Chitsaz, H. Shaker, H. Zareipour, D. Wood, and N. Amjady, "Shortterm electricity load forecasting of buildings in microgrids", Energy and Buildings, vol. 99, 2015, pp. 50-60.
Grazia Belli (Italy, 1985) received her degree in Energetic Engineering in 2011 and her Ph.D in Science of Complex Systems in 2016 from the University of Calabria. Her current research interests concern renewable energy sources, distributed generation, smart grid technologies and electricity local market.

Giovanni Brusco (Italy, 1980) received his degree in Electronics Engineering from the University of Calabria, Italy, in 2007 and his Ph.D. in Computer and system Engineering in 2013 at the Electronic, Computer and Systems Science Department of the University of Calabria, Italy. His current research interests concern renewable energy sources, distributed generation, harmonic analysis and smart grid technologies.

Alessandro Burgio (Italy, 1973) received his degree in Management Engineering from the University of Calabria in 1999 and his Ph.D. in Computer and system Engineering in 2006 at the Electronic, Computer and Systems Science Department of the University of Calabria, Italy. His current research interests include electrical power systems, distributed generation, renewable energy, power electronics and harmonics, electronic ballast.

Daniele Menniti (Italy 1958) received his degree in Electrical Engineering from the University of Calabria, Cosenza, Italy and his Ph.D. degree in Electrical Engineering from the University of Naples, Italy, in 1984 and 1989 respectively. He is an Associate Professor at the Mechanical, Energetic and Management Department of the University of Calabria, Italy. His current research interests concern electrical power system analysis, real-time control and automation.

Anna Pinnarelli (Italy, 1973) received her degree in Management Engineering from the University of Calabria in 1998 and her Ph.D. in Electrical Engineering in 2002 from the Electrical Engineering Department of the University of Naples, Italy. She is an Assistant Professor at the Mechanical, Energetic and Management Department of University of Calabria, Italy. Her current research interests concern FACTS technology, harmonic analysis, electrical system automation, decentralized control and smart grid technologies.

Nicola Sorrentino (Italy, 1970) received his degree in Management Engineering in 1994 and a Ph.D. in Computer and system Engineering in 1999 at the Electronic, Computer and Systems Science Department of the University of Calabria, Italy. $\mathrm{He}$ is a Researcher at the Mechanical, Energetic and Management Department of the University of Calabria, Italy.

Pasquale Vizza (Italy, 1990) received his degree in Energetic Engineering in 2014 from the University of Calabria; he is currently attending the $\mathrm{PhD}$ school at the same University. His current research interests include renewable energy sources, smart grid technologies, energy storage economics, generation and load forecasting. 